\title{
Modelling risk profiles of depression symptoms using Cloninger's temperament and character traits: a non- iterative approach to assess linear-by-linear association within ordered contingency tables
}

\author{
$\underline{\text { S. Zafar }}^{\mathrm{a}}$, I. L. Hudson ${ }^{\mathrm{a}}$, E. J. Beh ${ }^{\mathrm{a}}$, P. R. Joyce ${ }^{\mathrm{b}}$ \\ ${ }^{a}$ School of Mathematical and Physical Sciences, University of Newcastle, Newcastle, Australia. \\ ${ }^{b}$ Department of Psychological Medicine, Christchurch School of Medicine and Health Sciences, University \\ of Otago, New Zealand \\ Email: Sidra.Zafar@uon.edu.au
}

\begin{abstract}
Personality is linked to mental illness. The relationship between the seven temperament and character traits (TCIs), (Novelty seeking (NS), Harm Avoidance (HA), Reward Dependence (RD), Persistence (P), Self-directedness (S), Cooperativeness (C) and Self Transcendence (ST)), of Cloninger (1994) and three symptoms of psychological distress, or SCLs (Depression (D), Anxiety (A) and Psychoticism (Psy)) is investigated across gender and shown to have significantly different symptom profiles post treatment. The data used in this study was earlier analysed by Turner, et al. (2003) and comes from patients measured pre and post-treatment from the NZ Christchurch Psychotherapy of Depression Study (Joyce, et al. 2002). In this study we have used the newly developed direct estimation approach (Beh and Davy, 2004 and Zafar et al., 2015) to estimate the linear-by-linear association in two-way tables, within the framework of ordinal log-linear models (OLLMs), with the aim of analysing associations between the TCIs and SCLs. Two non-iterative estimators were considered for this study - the Beh-Davy non-iterative estimator (BDNI) (Beh and Davy, 2004) and the Log non-iterative estimator (LogNI) (Beh and Farver, 2009). The BDNI and LogNI estimation methods provide closed-form estimators which do not require iteration to estimate the linear-by-linear association parameter of OLLMs, unlike their conventional and iterative counter parts, such as the Newton-Raphson and the iterative proportional fitting methods.
\end{abstract}

The estimates obtained from the BDNI and LogNI estimation methods are reported, for pairwise relationships between TCIs and symptoms, along with the standard errors and p-values for males and females for pre and post treatment. Both estimators, BDNI and LogNI, provide estimates which are close to each other. We found significant changing relationships between the seven TCIs and psychological distress symptoms across gender for NS and P post treatment; with both TCIs and SCLs dichotomised by the median. We found statistically significant differences between the BDNI and LogNI estimates for males and females, post-treatment; establishing that higher levels of NS are associated with less D and Psy in males as compared to females. Higher HA is shown to be associated with higher D, A and Psy in males and females, pre and post-treatment. S is found to be negatively related to D, A and Psy for males and in females, pre and posttreatment. $\mathrm{P}$ is demonstrated as gender-specific only in the case of $\mathrm{D}$; with less $\mathrm{D}$ associated with higher levels of $\mathrm{P}$ in males comparison with females post treatment. In addition, we demonstrate the linear-by-linear association between pre-treatment TCI's and change in depression, anxiety and psychoticism $(\Delta \mathrm{D}, \Delta \mathrm{A}$ and $\Delta$ Psy), where the change is defined as categorised by the median scores of the (post - pre-treatment) levels. We show that pairwise association between three TCI's (HA, P and C) and two of our three symptoms of psychological distress, $\Delta \mathrm{D}$ and $\Delta \mathrm{Psy}$, are gender-specific. These results reported agree, in part, with preliminary univariate Generalised Additive Model for Location, Scale and Shape (GAMLSS) models based on the combined pre and post treatment data (Hudson et al., 2015a), which allow for non-linearity between TCIs and symptoms, and for interactions between TCI with gender and time.

Keywords: Ordinal log-linear model, non-iterative estimation, linear-by-linear association, temperament and character traits inventory (TCI), symptom checklist (SCL) 


\section{INTRODUCTION}

Ordinal log-linear models (OLLM's) are an extension of the well-known log-linear models for analysing the association between categorical variables that possess an inherent ordinal structure in a contingency table (Haberman, 1974). The procedures which are most frequently employed for estimating the parameters of OLLMs are iterative, namely, the Newton-Raphson algorithm and iterative proportional fitting; for details, see for example Agresti (1984, p. 237). However, Beh and Davy (2004) proposed a direct method for the estimation of the linear-by-linear association parameter. The non-iterative estimation methods are mainly based on orthogonal polynomials derived from the simple recurrence formulae of Emerson (1968) which have been used earlier to study association between ordered variables; see, for example, Rayner and Best, 1996. The two key non-iterative estimators, the Beh and Davy non-iterative estimator (BDNI) and the lognon-iterative estimator (LogNI), were shown to be accurate, stable and reliable estimators of the linear-bylinear association parameter by means of comparison with estimates using the Newton-Raphson method in several empirical and computational studies (Beh and Farver, 2009, 2012a,b). Zafar et al. (2013a) showed that the two non-iterative procedures, BDNI and LogNI, give unbiased estimates under the assumption of Poisson i.i.d cell frequencies. A recent application of the non-iterative estimation procedure to molecular drug discovery data was made by Zafar, et al. (2013b) who also considered the link between non-iterative estimation and the ordered correspondence analysis first described in Beh (1997). Furthermore, in order to enhance the applicability of non-iterative estimators, Zafar et al. (2015) recently explored, by way of mathematical methods, simulation and empirical studies, the bias, consistency, variance and the relative efficiency of BDNI and LogNI estimators by comparing them to the Cramer-Rao lower bound. Zafar et al. (2015) showed that the LogNI estimator is a Minimum Variance Unbiased (MVU) estimator of the linear-bylinear association parameter, under the assumption of independence.

The association between symptoms of psychological distress and personality traits is investigated in this study using the non-iterative estimation methods, BDNI and LogNI. The personality traits, measured pretreatment and post-treatment, are from the Temperament and Character Inventory (TCIs) developed by Cloninger et al. (1994) which operates with seven dimensions of personality traits. The four temperament traits are novelty seeking (NS), harm avoidance (HA), reward dependence (RD) and persistence (P) and the three character traits are self-directedness (SD), cooperativeness (C) and self-transcendence (ST). The data first analysed by Turner, et al. (2003) comes from patients measured pre and post treatment from the NZ Christchurch Psychotherapy of Depression Study (Joyce, et al. 2002). The Symptom Checklist (SCL) (Dergotis, 1983) has been used in this study which contains nine depression symptoms on patients, also measured pre-treatment and post-treatment, depicting the psychological distress of a person. The nine SCLs are somatisation (S), obsessive compulsive (OC), interpersonal sensitivity (IS), depression (D), anxiety (A), anger-hostility (AH), phobic anxiety (PA), paranoid ideation (PI) and psychoticism (Psy). Our study focuses on the bivariate relationships between the categorised variants of the seven TCIs and three of the nine SCLs (D, A and Psy). The aim of the study is to investigate swaps in sign of the pairwise associations across strata, where strata are defined by gender, for pre and post-treatment data. A brief review of the non-iterative estimation methods, BDNI and LogNI, and relevant literature is discussed in the following section. Section 3 describes the data and design used for the application of non-iterative estimation methods to examine the association between TCIs and SCLs. The results are discussed in section 4 followed by a discussion the last section.

\section{NON-ITERATIVE ESTIMATION METHODS}

For a doubly ordered $I \times J$ contingency table, $N$, denote the proportion of individuals/units in the $(i, j)$ th cell as $p_{i j}=n_{i j} / n$ where $n_{i j}$ is the $(i, j)$ th cell value of $N$, for $i=1,2, \ldots . I$, and $j=1,2, \ldots . . J$. Therefore $\sum_{i=1}^{I} \sum_{j=1}^{J} p_{i j}=1$. Denote $p_{i .}$ and $p_{. j}$ as the marginal proportion of the $i$ th row and $j$ th column categories, respectively, such that $\sum_{i=1}^{I} p_{i .}=\sum_{j=1}^{J} p_{. j}=1$. Moreover, let $m_{i j}$ be the expected cell frequency of the $(i, j)$ th cell, for example, when the row and column variables are independent, so that $\mathrm{m}_{\mathrm{ij}}=\mathrm{n}_{\mathrm{i} .} \mathrm{n}_{\mathrm{j}} / \mathrm{n}$. The well-known OLLM for a doubly ordered contingency table, as studied by Agresti $(1984$, p.76) and Beh and Farver (2009), is defined as

$$
\ln m_{i j}=\mu+\alpha_{i}+\beta_{j}+\varphi\left(u_{i}-\bar{u}\right)\left(v_{j}-\bar{v}\right)
$$

where $u_{i}$ and $v_{j}$ represent row and column scores for $i=1,2, \ldots, I$ and $j=1,2, \ldots, J$, respectively and are chosen a priori to reflect the ordered structure of each variable. The parameter $\mu$ is the grand mean of the expected cell frequencies and $\alpha_{i}$ and $\beta_{j}$ are the main effects of $i$ th row and $j$ th column, respectively. In our 
application, we choose the natural scores, $u_{i}=i$ and $v_{j}=j$, for the purpose of simplicity. The mean of the row scores is denoted by $\bar{u}=\sum_{i=1}^{I} p_{i} u_{i}$ while $\bar{v}=\sum_{j=1}^{J} p_{\text {.j }} v_{j}$ is the mean of the column scores. We also define $\sigma_{I}^{2}=\sum_{i=1}^{I} p_{i .} u_{i}^{2}-\bar{u}^{2}$ and $\sigma_{J}^{2}=\sum_{j=1}^{J} p_{. j} v_{j}^{2}-\bar{v}^{2}$. The grand mean effect, $\mu$, row effect, $\alpha_{i}$, and the column effect, $\beta_{j}$, parameters in (2.1), are estimated as: $\hat{\mu}=\ln n+\frac{1}{I} \sum_{i=1}^{I} \ln p_{i .}+\frac{1}{J} \sum_{j=1}^{J} \ln p_{. j}$, $\hat{\alpha}_{i}=\ln p_{i .}-\frac{1}{I} \sum_{i=1}^{I} \ln p_{i .}$ and $\hat{\beta}_{j}=\ln p_{. j}-\frac{1}{J} \sum_{j=1}^{J} \ln p_{. j}$. The parameter representing the linear-by-linear association, $\varphi$, in (2.1) can be interpreted in terms of the log-odds ratio, see for example, Ishi-Kuntz (1994) for a review on OLLM and respective details. Estimation of the parameter $\varphi$ in (2.1) can be performed using a number of iterative procedures - iterative proportional fitting and the Newton-Raphson procedure are the most commonly used. One may refer to, for example, the following for discussions on the OLLM (2.1), these iterative techniques and their use for the estimation of the parameters; Haberman (1974), Agresti (1984, Appendix B) and Beh and Farver (2009). Alternatively, the non-iterative estimation methods, BDNI and LogNI (Beh and Davy, 2004, Beh and Farver, 2009) can be used to estimate the linear-by-linear association parameter. The two key non-iterative estimators for the estimation of the parameter $\varphi$ are

$$
\hat{\varphi}_{L o g N I}=\frac{1}{\sigma_{I}^{2} \sigma_{J}^{2}} \sum_{i=1}^{I} \sum_{j=1}^{J} p_{i .} p_{. j}\left(u_{i}-\bar{u}\right)\left(v_{j}-\bar{v}\right) \ln \left(\frac{p_{i j}}{p_{i .} p_{. j}}\right)
$$

and

$$
\hat{\varphi}_{B D N I}=\frac{1}{\sigma_{I}^{2} \sigma_{J}^{2}} \sum_{i=1}^{I} \sum_{j=1}^{J} p_{i j}\left(u_{i}-\bar{u}\right)\left(v_{j}-\bar{v}\right) .
$$

The estimator (2.2) is referred to as the LogNI estimator due to the presence of the logarithm function. The second estimator (2.3), originally proposed by Beh and Davy (2004), is referred to as the BDNI estimator of $\varphi$. The non-iterative estimators of (2.2) and (2.3) are based on the first order orthogonal polynomials for the rows and columns, so that $\varphi$ reflects the linear-by-linear association between the ordered categorical variables. Rayner and Best (1996) used the same orthogonal polynomials to partition the chi-squared statistic into linear, quadratic and higher order components. Many studies have also demonstrated the use of such orthogonal polynomials for graphically displaying the association between the variables of a contingency tables using correspondence analysis; see Beh and Lombardo (2014) for an extensive discussion on this topic. It can be seen that the estimator (2.3) is obtained by the first order Taylor series expansion $\ln \left(\mathrm{p}_{\mathrm{ij}} / \mathrm{p}_{\mathrm{i} . \mathrm{p}} \mathrm{p}_{\mathrm{j}}\right) \approx$ $\mathrm{p}_{\mathrm{ij}} / \mathrm{p}_{\mathrm{i} . \mathrm{p} . \mathrm{j}}-1$. The performance of (2.2) and (2.3), in comparison with the estimator obtained from the more traditional Newton - Raphson approach, was extensively studied by Beh and Farver (2012a,b) in a series of computational studies. A link between the LogNI and BDNI estimators was established by using the BoxCox transformation; see Beh and Farver (2012c).

\section{DATA AND DESIGN}

The data analysed was based on combining two datasets from the Christchurch Outcome of Depression Study and Christchurch Psychotherapy of Depression Study (Joyce et al. 2002). The first was a randomised controlled trial (RCT) of two antidepressants (fluoxetine or nortriptyline), the second a RCT of two psychotherapies (interpersonal psychotherapy and cognitive therapy). There was a total of 346 patients (124 males, 222 females) pre-treatment, and 202 patients (67 males, 135 females) post-treatment. The relationship between personality and character traits and the symptoms of psychological distress is investigated by considering the linear-by-linear association between the seven TCI's, developed by Cloninger et al. (1994) and three of the nine SCLS's (D, A and Psy) (Derogatis, 1983). Each TCI and SCL symptom was dichotomised above and below the median for that symptom (separately for pre and post-treatment); resulting in $2 \times 2$ contingency tables for pairwise relationships between TCI and SCL, where each TCI and SCL has two levels. High SCL level ( $>$ median) indicate increased symptoms, and higher level of the continuous TCI relate to elevated TCI trait. We also define the three (post - pre-treatment) SCL's $(\Delta \mathrm{D}, \Delta \mathrm{A}$ and $\Delta$ Psy) and investigate their relationship with pre-treatment TCI's for 202 patients to gain insight into the gender differences across treatment.

\section{RESULTS}

Table 1, 2, 3 and 4 show the calculated BDNI and LogNI estimates for pairwise relationships between TCIs and symptoms (D, A and Psy), along with their standard errors and p-values for males and females for pre and post treatment. The standard errors (SE) for BDNI and the LogNI estimators are calculated by the formulas given by Zafar et al. (2015, section 4). From Tables 1 to 6 , it can be seen that $\hat{\varphi}_{B D N I}$ and $\hat{\varphi}_{\text {LogNI }}$, given by (2.2) and (2.3), provide estimates which are close to each other. The BDNI estimator underestimates 
the linear-by-linear association; confirming the findings of computational studies carried out by Beh and Farver (2012a, b, Zafar et al., 2013a, 2015). However, earlier Beh and Farver (2012a,b) showed using the Wald test that the BDNI and LogNI estimates are statistically similar to the estimates obtained by the Newton - Raphson method. Therefore, either the non-iterative estimator, BDNI or LogNI, can be used for quantifying the linear-by-linear association. For demonstration purposes, we present the results for both BDNI and LogNI estimators. For Table 1-6, the statistically significant pairwise associations at $5 \%$ level of significance are bolded and significant gender differences at the $5 \%$ level of significance are both bolded and shaded.

Table 1: The $\hat{\varphi}_{B D N I}$ for pairwise relationships between TCIs and SCLs (D, A and Psy) for males and females (pre-treatment)

\begin{tabular}{|c|c|c|c|c|c|c|}
\hline & \multicolumn{2}{|c|}{$\mathrm{D}$} & \multicolumn{2}{|c|}{ A } & \multicolumn{2}{|c|}{ Psy } \\
\hline & $\begin{array}{c}\text { Males } \\
\hat{\varphi}_{B D N I}(\mathrm{SE})[\mathrm{p} \text {-value }]\end{array}$ & $\begin{array}{c}\text { Females } \\
\hat{\varphi}_{B D N I}(\mathrm{SE})[\mathrm{p} \text {-value] }\end{array}$ & $\begin{array}{c}\text { Males } \\
\hat{\varphi}_{B D N I}(\mathrm{SE})[\mathrm{p} \text {-value] } \\
\end{array}$ & $\begin{array}{c}\text { Females } \\
\hat{\varphi}_{B D N I}(\mathrm{SE})[\mathrm{p} \text {-value] }\end{array}$ & $\begin{array}{c}\text { Males } \\
\hat{\varphi}_{B D N I}(\mathrm{SE})[\mathrm{p} \text {-value }]\end{array}$ & $\begin{array}{c}\text { Females } \\
\hat{\varphi}_{B D N I}(\mathrm{SE})[\mathrm{p} \text {-value] } \\
\end{array}$ \\
\hline NS & $-0.50(0.36)[0.08]$ & $-0.14(0.22)[0.26]$ & $-0.32(0.36)[0.19]$ & $-0.12(0.22)[0.29]$ & $0.02(0.36)[0.48]$ & $0.25(0.22)[0.12]$ \\
\hline HA & $0.82 *(0.37)[0.01]$ & $0.91 *(0.22)[0.00]$ & $0.72 *(0.36)[0.02]$ & $0.60 *(0.22)[0.00]$ & $0.54(0.36)[0.07]$ & $0.70 *(0.22)[0.00]$ \\
\hline $\mathrm{RD}$ & $-0.10(0.39)[0.40]$ & $-0.22(0.22)[0.16]$ & $0.48(0.39)[0.11]$ & $-0.10(0.22)[0.33]$ & $0.19(0.39)[0.31]$ & $-0.24(0.22)[0.13]$ \\
\hline$P$ & $0.48(0.36)[0.09]$ & $-0.02(0.22)[0.46]$ & $0.19(0.36)[0.30]$ & $0.19(0.22)[0.20]$ & $0.12(0.36)[0.37]$ & $-0.23(0.22)[0.40]$ \\
\hline $\mathrm{S}$ & $-1.00 *(0.36)[0.00]$ & $-1.28 *(0.22)[0.00]$ & $-0.91 *(0.36)[0.01]$ & $-1.11 *(0.22)[0.00]$ & $-1.09 *(0.36)[0.00]$ & $-1.44 *(0.22)[0.00]$ \\
\hline $\mathrm{C}$ & $-0.43(0.38)[0.13]$ & $-0.44 *(0.22)[0.02]$ & $-0.63(0.38)[0.05]$ & $-0.46 *(0.22)[0.02]$ & $-1.35 *(0.39)[0.00]$ & $-0.84 *(0.22)[0.00]$ \\
\hline ST & $0.37(0.36)[0.15]$ & $0.53 *(0.22)[0.01]$ & $1.23 *(0.36)[0.00]$ & $0.79 *(0.22)[0.00]$ & $1.15 *(0.36)[0.00]$ & $0.79 *(0.22)[0.000]$ \\
\hline
\end{tabular}

Table 2: The $\hat{\varphi}_{\text {LogNI }}$ for pairwise relationships between TCIs and SCLs (D, A and Psy) for males and females (pre-treatment)

\begin{tabular}{|c|c|c|c|c|c|c|}
\hline & \multicolumn{2}{|c|}{$\mathrm{D}$} & \multicolumn{2}{|c|}{ A } & \multicolumn{2}{|c|}{ Psy } \\
\hline & $\begin{array}{c}\text { Males } \\
\hat{\varphi}_{\text {LogNI }}(\mathrm{SE})[\mathrm{p} \text {-value }]\end{array}$ & $\begin{array}{c}\text { Females } \\
\hat{\varphi}_{\text {LogNI }}(\mathrm{SE})[\mathrm{p} \text {-value] }\end{array}$ & $\begin{array}{c}\text { Males } \\
\hat{\varphi}_{\text {LogNI }} \text { (SE)[p-value] }\end{array}$ & $\begin{array}{c}\text { Females } \\
\hat{\varphi}_{\text {LogNI }}(\mathrm{SE})[\mathrm{p} \text {-value }]\end{array}$ & $\begin{array}{c}\text { Males } \\
\hat{\varphi}_{\text {Log } N I}(\mathrm{SE})[\mathrm{p} \text {-value] }\end{array}$ & $\begin{array}{c}\text { Females } \\
\hat{\varphi}_{\text {LogNI }}(\mathrm{SE})[\mathrm{p} \text {-value }\end{array}$ \\
\hline NS & $-0.51(0.37)[0.09]$ & $-0.14(0.22)[0.26]$ & $-0.32(0.36)[0.19]$ & $-0.12(0.22)[0.29]$ & $0.02(0.36)[0.48]$ & $0.26(0.22)[0.12]$ \\
\hline HA & $0.83 *(0.37)[0.01]$ & $0.92 *(0.22)[0.00]$ & $0.73 *(0.37)[0.02]$ & $0.61 *(0.22)[0.00]$ & $0.54(0.37)[0.07]$ & $0.71 *(0.22)[0.00]$ \\
\hline $\mathrm{RD}$ & $-0.10(0.39)[0.40]$ & $-0.22(0.22)[0.16]$ & $0.48(0.39)[0.11]$ & $-0.10(0.22)[0.33]$ & $0.19(0.39)[0.31]$ & $-0.24(0.22)[0.13]$ \\
\hline$P$ & $0.48(0.37)[0.10]$ & $-0.02(0.22)[0.46]$ & $0.19(0.36)[0.30]$ & $0.19(0.22)[0.19]$ & $0.12(0.36)[0.37]$ & $-0.23(0.22)[0.15]$ \\
\hline $\mathrm{S}$ & $-1.04 *(0.38)[0.00]$ & $-1.33 *(0.23)[0.00]$ & $-0.92 *(0.37)[0.01]$ & $-1.14 *(0.22)[0.00]$ & $-1.11 *(0.38)[0.00]$ & $-1.51 *(0.23)[0.00]$ \\
\hline $\mathrm{C}$ & $-0.44(0.39)[0.13]$ & $-0.44 *(0.22)[0.02]$ & $-0.63(0.39)[0.05]$ & $-0.47 *(0.22)[0.02]$ & $-1.41 *(0.41)[0.00]$ & $-0.85 *(0.22)[0.00]$ \\
\hline ST & $0.37(0.37)[0.16]$ & $0.59 *(0.22)[0.00]$ & $1.27 *(0.38)[0.00]$ & $0.80 *(0.22)[0.00]$ & $1.19 *(0.38)[0.00]$ & $0.80 *(0.22)[0.00]$ \\
\hline
\end{tabular}

From Tables 1-4, NS has insignificant relationships with all symptoms of psychological distress for males and females, pre-treatment. For males, post-treatment, NS is significantly negatively related to D, A and Psy. Furthermore, NS is gender specific for D as the linear-by-linear association is negative for males and positive for females, post-treatment [difference between the BDNI estimates for males and females is statistically significant at 5\%]. From Table 3 and 4, for the relationship between NS and Psy, indicates the difference between the magnitude of BDNI estimates for males and females is likewise statistically significant; implying that males are less depressed in comparison with females with increasing NS. In general, HA is statistically significantly positively related to all symptoms of psychological distress, pre and post-treatment, and is not gender-specific; implying that higher levels of HA are associated with higher depression in both genders. No significant relationships were observed among RD and distress symptoms for males and females, pre-treatment. However, RD is significantly negatively associated with D and A for males, post-treatment. Post-treatment, $\mathrm{P}$ is gender-specific for $\mathrm{D}$ with the difference between BDNI estimates for males and females being statistically significant. More specifically, for males, post-treatment, $\mathrm{P}$ is significantly negatively related to $\mathrm{D}$. On the contrary, for females, $\mathrm{P}$ is positively related to $\mathrm{D}$. No significant relationships were observed between $\mathrm{P}$ and symptoms for males and females, pre-treatment. In general, $\mathrm{S}$ is significantly negatively related to all SCL implying that the higher the $\mathrm{S}$, the less depressed patients are; however, $\mathrm{S}$ is not gender-specific pre and post-treatment. For males, pre-treatment, $\mathrm{C}$ is significantly negatively related to Psy and significantly negatively related $\mathrm{A}$ and Psy post-treatment. For females, pre and post-treatment, $\mathrm{C}$ is significantly negatively associated to D, A and Psy, with the exception of the association between $\mathrm{C}$ and $\mathrm{A}$, post-treatment, where the relationship is insignificant. In the case of ST, ST is positively related to all three symptoms of distress, pre and post-treatment, and is only gender-specific for $\mathrm{D}$ (post-treatment). All relationships among ST and distress symptoms are significant pre-treatment, for males and females, except 
ST and D for males. In summary, NS and P are found to be gender-specific for the three symptoms of psychological distress.

Table 3: The $\hat{\varphi}_{B D N I}$ for pairwise relationships between TCIs and SCLs (D, A and Psy) for males and females (post-treatment)

\begin{tabular}{|c|c|c|c|c|c|c|}
\hline & \multicolumn{2}{|c|}{$\mathrm{D}$} & \multicolumn{2}{|c|}{$\mathrm{A}$} & \multicolumn{2}{|c|}{ Psy } \\
\hline & $\begin{array}{c}\text { Males } \\
\hat{\varphi}_{B D N I}(\mathrm{SE})[\mathrm{p} \text {-value }]\end{array}$ & $\begin{array}{c}\text { Females } \\
\hat{\varphi}_{B D N I}(\mathrm{SE})[\mathrm{p} \text {-value }]\end{array}$ & $\begin{array}{c}\text { Males } \\
\hat{\varphi}_{B D N I}(\mathrm{SE})[\mathrm{p} \text {-value }]\end{array}$ & $\begin{array}{c}\text { Females } \\
\hat{\varphi}_{B D N I}(\mathrm{SE})[\mathrm{p}-\mathrm{value}]\end{array}$ & $\begin{array}{c}\text { Males } \\
\hat{\varphi}_{B D N I}(\mathrm{SE})[\mathrm{p} \text {-value }]\end{array}$ & $\begin{array}{c}\text { Females } \\
\hat{\varphi}_{B D N I}(\mathrm{SE})[\mathrm{p}-\mathrm{v} \text { alue }]\end{array}$ \\
\hline NS & $-1.71 *(0.50)[0.00]$ & $0.02(0.36)[0.47]$ & $-0.93 *(0.50)[0.03]$ & $-0.37(0.36)[0.14]$ & $-1.41 *(0.51)[0.00]$ & $-0.04(0.36)[0.46]$ \\
\hline HA & $1.87 *(0.50)[0.00]$ & $1.58 *(0.36)[0.00]$ & $1.32 *(0.49)[0.00]$ & $1.35 *(0.36)[0.00]$ & $1.32 *(0.51)[0.00]$ & $1.38 *(0.35)[0.00]$ \\
\hline $\mathrm{RD}$ & $-1.58 *(0.57)[0.00]$ & $-0.69 *(0.37)[0.03]$ & $-1.62 *(0.55)[0.00]$ & $-0.68 *(0.37)[0.03]$ & $-0.74(0.57)[0.10]$ & $-0.50(0.36)[0.09]$ \\
\hline $\mathrm{C}$ & $-0.83(0.56)[0.07]$ & $-1.00 *(0.37)[0.00]$ & $-1.49 *(0.56)[0.00]$ & $-0.46(0.37)[0.11]$ & $-1.47 *(0.60)[0.00]$ & $-0.81 *(0.37)[0.01]$ \\
\hline ST & $-0.11(0.50)[0.42]$ & $-0.06(0.36)[0.44]$ & $0.65(0.50)[0.10]$ & $0.59(0.36)[0.05]$ & $0.00(0.52)[0.50]$ & $0.51(0.38)[0.09]$ \\
\hline
\end{tabular}

${ }^{*}$ statistically significant at $5 \%$ level of significance

Table 4: The $\hat{\varphi}_{\text {LognI }}$ for pairwise relationships between TCIs and SCLs (D, A and Psy) for males and females (post-treatment)

\begin{tabular}{|c|c|c|c|c|c|c|}
\hline & \multicolumn{2}{|c|}{$\mathrm{D}$} & \multicolumn{2}{|c|}{ A } & \multicolumn{2}{|c|}{ Psy } \\
\hline & $\begin{array}{c}\text { Males } \\
\hat{\varphi}_{\text {LogNI }}(\mathrm{SE})[\mathrm{p} \text {-value }]\end{array}$ & $\begin{array}{c}\text { Females } \\
\hat{\varphi}_{\text {LogNI }}(\mathrm{SE})[\mathrm{p} \text {-value }]\end{array}$ & $\begin{array}{c}\text { Males } \\
\hat{\varphi}_{\text {LogNI }}(\mathrm{SE})[\mathrm{p} \text {-value] }\end{array}$ & $\begin{array}{c}\text { Females } \\
\hat{\varphi}_{\text {LogNI }}(\mathrm{SE})[\mathrm{p} \text {-value }]\end{array}$ & $\begin{array}{c}\text { Males } \\
\hat{\varphi}_{\text {LogNI }} \text { (SE)[p-value] }\end{array}$ & $\begin{array}{c}\text { Females } \\
\hat{\varphi}_{\text {LogNI }} \text { (SE) }[\mathrm{p} \text {-value] }\end{array}$ \\
\hline NS & $-1.86 *(0.57)[0.00]$ & $0.02(0.36)[0.47]$ & $-0.95 *(0.51)[0.03]$ & $-0.37(0.36)[0.16]$ & $-1.50 *(0.57)[0.00]$ & $-0.04(0.36)[0.46]$ \\
\hline $\mathrm{HA}$ & $2.09 *(0.60)[0.00]$ & $1.71 *(0.40)[0.00]$ & $1.39 *(0.53)[0.00]$ & $1.43 *(0.39)[0.00]$ & $1.41 *(0.57)[0.00]$ & $1.46 *(0.39)[0.00]$ \\
\hline $\mathrm{RD}$ & $-1.57 *(0.56)[0.00]$ & $-0.69 *(0.36)[0.03]$ & $-1.66 *(0.56)[0.00]$ & $-0.68 *(0.37)[0.03]$ & $-0.72(0.54)[0.09]$ & $-0.50(0.36)[0.08]$ \\
\hline $\mathrm{C}$ & $-0.82(0.54)[0.06]$ & $-1.01 *(0.37)[0.00]$ & $-1.52 *(0.56)[0.00]$ & $-0.46(0.36)[0.10]$ & $-1.45 *(0.56)[0.00]$ & $-0.81 *(0.36)[0.01]$ \\
\hline ST & $-0.11(0.50)[0.42]$ & $-0.06(0.36)[0.44]$ & $0.65(0.50)[0.10]$ & $0.59(0.36)[0.05]$ & $0.00(0.52)[0.50]$ & $0.52(0.36)[0.08]$ \\
\hline
\end{tabular}

Table 5 and 6 give the BDNI and LogNI estimates for males and females, respectively, for pairwise associations among pre TCI's and $\Delta \mathrm{D}, \Delta \mathrm{A}$ and $\Delta \mathrm{Psy}$. From Table 5, NS is not statistically significantly related to any symptom of distress for either gender. For males, in general, $\mathrm{HA}$ is positively related to $\Delta \mathrm{D}, \Delta \mathrm{A}$ and $\Delta$ Psy; however, only the linear-by-linear associations between $\mathrm{HA}$ and $\Delta \mathrm{D}$, and $\mathrm{HA}$ and $\Delta \mathrm{A}$ are statistically significant implying that the higher the level of HA, the less patients were depressed post treatment. For HA and $\Delta \mathrm{D}$, the difference between the magnitude of the BDNI estimates across the sexes is significant at $5 \%$ significance level which suggests that males are less depressed compared to females for higher HA. No gender differences were observed for RD and the symptoms of psychological distress. For males, RD is significantly negatively related to $\Delta \mathrm{D}$ and $\Delta \mathrm{D}$ suggesting that as $\mathrm{RD}$ increases, the males become more depressed. It is also observed that $\mathrm{P}$ is gender-specific in the case of $\Delta \mathrm{Psy}$, with males less depressed than females as $\mathrm{P}$ increases.

Table 5: The $\hat{\varphi}_{B D N I}$ for pairwise relationships between Pre-treatment TCIs and SCL $(\Delta \mathrm{D}, \Delta \mathrm{A}$ and $\Delta$ Psy, where $\Delta=$ Post - Pre) for males and females

\begin{tabular}{|c|c|c|c|c|c|c|}
\hline & \multicolumn{2}{|c|}{$\Delta \mathrm{D}$} & \multicolumn{2}{|c|}{$\Delta \mathrm{A}$} & \multicolumn{2}{|c|}{$\Delta$ Psy } \\
\hline & $\begin{array}{c}\text { Males } \\
\hat{\varphi}_{B D N I} \text { (SE)[p-value] }\end{array}$ & $\begin{array}{c}\text { Females } \\
\hat{\varphi}_{B D N I}(\mathrm{SE})[\mathrm{p} \text {-value] }\end{array}$ & $\begin{array}{c}\text { Males } \\
\hat{\varphi}_{B D N I} \text { (SE)[p-value] }\end{array}$ & $\begin{array}{c}\text { Females } \\
\hat{\varphi}_{B D N I} \text { (SE)[p-value] }\end{array}$ & $\begin{array}{c}\text { Males } \\
\hat{\varphi}_{B D N I}(\mathrm{SE})[\mathrm{p} \text {-value] }\end{array}$ & $\begin{array}{c}\text { Females } \\
\hat{\varphi}_{B D N I}(\mathrm{SE})[\mathrm{p} \text {-value }]\end{array}$ \\
\hline NS & $-0.63(0.50)[0.10]$ & $0.30(0.35)[0.19]$ & $-0.35(0.49)[0.24]$ & $-0.01(0.35)[0.49]$ & $-0.57(0.50)[0.13]$ & $0.047 * *(0.35)[0.09]$ \\
\hline HA & $1.53 * *(0.49)[0.00]$ & $0.16(0.35)[0.32]$ & $0.89 *(0.49)[0.04]$ & $-0.14(0.35)[0.34]$ & $0.78 * *(0.51)[0.06]$ & $0.16(0.35)[0.32]$ \\
\hline $\mathrm{RD}$ & $-0.99 *(0.53)[0.03]$ & $-0.01(0.35)[0.49]$ & $-0.88 *(0.51)[0.04]$ & $0.03(0.35)[0.47]$ & $0.12(0.54)[0.41]$ & $0.44(0.35)[0.10]$ \\
\hline $\mathrm{P}$ & $0.27(0.49)[0.29]$ & $-0.11(0.35)[0.37]$ & $0.21(0.49)[0.33]$ & $-0.16(0.35)[0.32]$ & $1.43 *(0.50)[0.00]$ & $-0.10(0.35)[0.38]$ \\
\hline $\mathrm{S}$ & $-0.50(0.50)[0.16]$ & $0.62 *(0.35)[0.04]$ & $-0.49(0.49)[0.16]$ & $0.54 * *(0.35)[0.06]$ & $0.31(0.51)[0.28]$ & $1.07 *(0.35)[0.00]$ \\
\hline $\mathrm{C}$ & $-1.14 *(0.53)[0.02]$ & $0.13(0.35)[0.35]$ & $0.04(0.52)[0.47]$ & $-0.46(0.35)[0.10]$ & $-0.06(0.54)[0.46]$ & $0.33(0.35)[0.17]$ \\
\hline ST & $0.03(0.49)[0.48]$ & $-0.48^{* * *}(0.35)[0.09]$ & $-0.27(0.49)[0.29]$ & $-0.64 *(0.35)[0.03]$ & $0.15(0.51)[0.39]$ & $-0.58 * *(0.35)[0.05]$ \\
\hline
\end{tabular}

For females, the relationship between $\mathrm{S}$ and $\Delta \mathrm{D}$, and $\mathrm{S}$ and $\Delta \mathrm{Psy}$ is significantly positive, implying that with increasing $\mathrm{S}$, depression improves. However, a gender difference is not found between $\mathrm{S}$ and all of $\Delta \mathrm{D}, \Delta \mathrm{A}$ and $\Delta$ Psy. Moreover, $\mathrm{C}$ is significantly gender-specific for $\Delta \mathrm{D}$, suggesting that males are more depressed 
with higher $\mathrm{C}$ pre-treatment. For males, no significant relationships are observed between ST and the symptoms of distress. For females, $\mathrm{ST}$ is statistically significantly negatively related $\Delta \mathrm{D}, \Delta \mathrm{A}$ and $\Delta$ Psy, which indicate that the females are more depressed after treatment for increased pre-treatment ST. In brief baseline $\mathrm{HA}$ and $\mathrm{C}$ had gender-specific effects on improvement of depression and Psychoticism $(\Delta \mathrm{A}$ and $\Delta$ Psy).

Table 6: The $\hat{\varphi}_{L o g N I}$ for pairwise relationships between Pre-treatment TCIs and SCL $(\Delta \mathrm{D}, \Delta \mathrm{A}$ and $\Delta$ Psy, where $\Delta=$ Post - Pre) for males and females

\begin{tabular}{|c|c|c|c|c|c|c|}
\hline & \multicolumn{2}{|c|}{$\Delta \mathrm{D}$} & \multicolumn{2}{|c|}{$\Delta \mathrm{A}$} & \multicolumn{2}{|c|}{$\Delta$ Psy } \\
\hline & $\begin{array}{c}\text { Males } \\
\hat{\varphi}_{\text {LogNI }} \text { (SE)[p-value] }\end{array}$ & $\begin{array}{c}\text { Females } \\
\hat{\varphi}_{\text {LogNI }} \text { (SE)[p-value] }\end{array}$ & $\begin{array}{c}\text { Males } \\
\hat{\varphi}_{\text {LogNI }} \text { (SE)[p-value] }\end{array}$ & $\begin{array}{c}\text { Females } \\
\hat{\varphi}_{\text {LogNI }} \text { (SE)[p-value] }\end{array}$ & $\begin{array}{c}\text { Males } \\
\hat{\varphi}_{\text {LogNI }} \text { (SE)[p-value] }\end{array}$ & $\begin{array}{c}\text { Females } \\
\hat{\varphi}_{\text {LogNI }} \text { (SE)[p-value] }\end{array}$ \\
\hline NS & $-0.63(0.50)[0.10]$ & $0.30(0.35)[0.19]$ & $-0.35(0.50)[0.24]$ & $-0.01(0.35)[0.49]$ & $-0.58(0.52)[0.13]$ & $0.047(0.35)[0.45]$ \\
\hline HA & $1.63 *(0.54)[0.00]$ & $0.16(0.35)[0.32]$ & $0.90 *(0.50)[0.04]$ & $-0.14(0.35)[0.34]$ & $0.79 * *(0.52)[0.06]$ & $0.16(0.35)[0.33]$ \\
\hline $\mathrm{RD}$ & $-1.00 *(0.53)[0.03]$ & $-0.01(0.35)[0.49]$ & $-0.92 * *(0.54)[0.05]$ & $0.03(0.35)[0.47]$ & $0.12(0.53)[0.41]$ & $0.45(0.35)[0.10]$ \\
\hline $\mathrm{P}$ & $0.27(0.49)[0.29]$ & $-0.11(0.35)[0.37]$ & $0.21(0.49)[0.33]$ & $-0.16(0.35)[0.32]$ & $1.54 *(0.56)[0.00]$ & $-0.10(0.35)[0.38]$ \\
\hline $\mathrm{S}$ & $-0.50(0.50)[0.16]$ & $0.62 *(0.35)[0.04]$ & $-0.49(0.50)[0.16]$ & $0.54 * *(0.35)[0.06]$ & $0.31(0.51)[0.27]$ & $1.10 *(0.36)[0.00]$ \\
\hline $\mathrm{C}$ & $-1.15 *(0.54)[0.00]$ & $0.13(0.35)[0.35]$ & $0.04(0.52)[0.47]$ & $-0.46(0.35)[0.10]$ & $-0.06(0.54)[0.45]$ & $0.34(0.35)[0.17]$ \\
\hline ST & $0.03(0.49)[0.48]$ & $-0.48 * *(0.35)[0.09]$ & $-0.27(0.49)[0.29]$ & $-0.65 *(0.35)[0.03]$ & $0.15(0.51)[0.39]$ & $-0.58^{* *}(0.35)[0.05]$ \\
\hline
\end{tabular}

\section{DISCUSSION AND CONCLUSIONS}

The study presented in this paper considers two non-iterative estimation methods, BDNI and LogNI (Beh and Davy, 2004, Beh and Farver, 2009) for examining the bivariate association between all seven personality and character traits, and three symptoms of distress, for both males and females for pre and post-treatment. In general, the BDNI and LogNI estimates exhibit similar behaviour as compared to the previous empirical and computational studies. Whilst both are accurate, the BDNI estimator underestimates the linear-by-linear association for all examined pairwise relationships between TCIs and SCLs.

We demonstrate that NS and P are gender-specific, post-treatment, with statistically significant differences between the BDNI and LogNI estimates across gender post-treatment; indicating that higher levels of NS are associated with lower depression in males as compared to females. Higher levels of HA are shown to be associated with higher depression in both sexes, pre and post-treatment. For males, less depression is associated with higher levels of $\mathrm{P}$ in comparison with females post-treatment; thereby establishing that $\mathrm{P}$ is gender-specific for D. For males and females, pre and post-treatment, higher $\mathrm{S}$ is associated with less depression in agreement with Hudson et al., (2015a,b), Leigh et al., 2012, Lee et al,, (2012). In addition, the pairwise relationships between $\mathrm{HA}, \mathrm{P}$ and $\mathrm{C}$, with $\Delta \mathrm{D}$ and $\Delta \mathrm{Psy}$ are shown to be gender-specific. In summary, higher HA and $\mathrm{P}$ are associated with improved depression in males, and higher levels of $\mathrm{C}$ are associated with less improvement in depression in the males. In summary for females more improvement in depression post treatment for increased baseline C. Likewise males exhibit more improvement in depression and anxiety post treatment for increased baseline HA compared to females. Whereas males exhibit less improvement for depression post treatment for increased baseline RD than females and likewise with increased baseline $\mathrm{C}$, the female trends are opposite. The males improve on psychoticism for higher baseline P. For females more increased baseline ST is associated with higher, and therefore, less improvement in depression, anxiety and psychoticism post treatment, not so for males. These results reported agree, in part, with preliminary univariate Generalised Additive Model for Location, Scale and Shape (GAMLSS) models based on the combined pre and post treatment data (Hudson et al., 2015a), which allow for non-linearity between TCIs and symptoms, and for interactions between TCI with gender and time. The non-iterative estimation methods described in our study will also enable the analyst to explore the quadratic and higher order associations in contingency tables with ordered variables. Future research will apply these extensions to quantify quadratic relationships between the personality and character traits and symptoms of psychological distress.

\section{REFERENCES}

Agresti, A. (1984), Categorical Data Analysis, New York: Wiley.

Beh, E.J. (1997). Simple correspondence analysis of ordinal cross-classifications using orthogonal polynomials, Biometrical Journal, 39, 589-613. 
Beh, E.J. \& Davy, P.J. (2004). A non-iterative alternative to ordinal log-linear models, Journal of Applied Mathematics \& Decision Sciences, 7(2), 1-20.

Beh, E.J. \& Farver, T.B. (2009). an evaluation of non-iterative methods for estimating the linear-by-linear parameter for ordinal log-linear models, Australian \& New Zealand Journal of Statistics, 51(3), 335-352.

Beh, E.J. \& Farver, T.B. (2012a). A computational study assessing maximum likelihood and non-iterative methods for estimating the linear-by-linear parameter for ordinal log-linear models, ISRN Comp. Math. doi: $10.5402 / 2012 / 396831,8$ pages.

Beh, E.J. and Farver, T.B. (2012b). On estimating the linear-by-linear parameter for ordinal log-linear models: a computational study. ISRN Computational Mathematics, 12 pages (doi:10.5402/2012/340415).

Beh, E.J. and Farver, T.B. (2012c). The Box-Cox transformation and non-iterative estimation methods for ordinal log-linear models. The Australian and New Zealand Journal of Statistics 54(4), 475 - 484.

Beh, E. J. and Lombardo, R. (2014). Correspondence Analysis: Theory, Practice and New Strategies, Chichester: Wiley.

Cloninger, C., Przybeck, T., Svrakic, D. and Wetzel, R. (1994). The Temperament and Character Trait Inventory (TCI): A Guide to Its Development and Use. Center for Psychobiology and Personality, Washington University, St. Louis, Missouri.

Derogatis, L.R. (1983) SCL-90-R, Administration, scoring and procedures Manual II for the Revised Version of the SCL-90. Baltimore: John Hopkins University Press.

Emerson, P.L. (1968). Numerical construction of orthogonal polynomials from a general recurrence formula, Biometrics, 24(3), 695-701.

Haberman, S.J. (1974a). Log-linear models for frequency tables with ordered classifications. Biometrics, 36, $589-600$.

Hudson, I.L., Turner, R.M. and Joyce, P.R. (2015a). Gender specific prediction of depression symptoms using Cloninger's personality traits: a GAMLSS approach. (in prep).

Hudson, I.L., Leigh, L., Joyce, P.R. (2015b). Modelling change in multivariate depression symptoms adjusting for gender and baseline temperament and character traits: a Latent Transition approach. 21st International Congress on Modelling and Simulation (MODSIM2015), Gold Coast, 29 Nov-Dec 4 (7 pages).

Joyce, P.R., Mulder, R.T., Luty, S.E., Sullivan, P.F., McKenzie, J.M., Abbott, R.M., and Stevens, I.F. (2002). Patterns and predictors of remission, response and recovery in major depression treated with fluoxetine or nortriptyline. Australian and New Zealand Journal of Psychiatry, 36,384-391.

Lee, S., Hudson, I.L., Lee, S., Turner, R. (2012). Visualizing improved prognosis in psychiatric treatment via mixtures, SOMS and Chernoff faces. Australian Statistical Conference. Statistical Society of Australia, pp131, 9 - 12 July 2012 Adelaide, SA.

Leigh, L., Hudson, I.L., Turner, R. and Joyce, P. (2012). Classifying multivariate risk profiles of depression symptoms using Cloninger's temperament and character traits: A latent class model approach. Australian Statistical Conference. Statistical Society of Australia, pp 132, 9-12 July 2012. Adelaide, SA.

Rayner, J.C.W. and Best, D.J. (1996), Smooth extensions of Pearson's product moment correlation and Spearman's rho. Statistics \& Probability Letters, 30, 171 - 177.

Turner, R.M., Hudson I.L., Butler, P.H. and Joyce, P.R. (2003). Brain Function and personality in normal males: a SPECT study using statistical parametric mapping. NeuroImage, 19, 1145-1162.

Zafar, S., Cheema, S. A., Beh, E. J. and Hudson, I. L. (2013a), A study of bias for non-iterative estimates of the linear-by-linear association parameter from the ordinal log-linear model, Advances on Latent Variables - Methods, Models and Applications, ISBN 97888343 2556, 6 pages.

Zafar, S., Cheema, S.A., Beh, E.J. and Hudson, I.L. (2013b). Linking ordinal log-linear models with Correspondence Analysis: an application to estimating drug-likeness in the drug discovery process. In Piantadosi, J., Anderssen, R.S. and Boland J. (eds) MODSIM 2013. $20^{\text {th }}$ International Congress on Modelling and Simulation Society of Australia and New Zealand. December 2013. 1941-1951. ISBN: 9780-9872143-3-1. www.mssanz.org.au/modsim2013/I1/zafar.pdf.

Zafar, S., Cheema, S.A., Beh, E.J. and Hudson, I.L. (2015). Properties of non-iterative estimators of the linear-by-linear association parameter of log-linear models for ordinal variables. Submitted. 